\title{
Avaliação do músculo tibial anterior de ratos submetidos a um modelo de artrite reumatoide $e$ tratados com laser de baixa potência
}

\section{Evaluation of the tibialis anterior muscle of rats submitted to a model of rheumatoid arthritis and treated with low-power laser}

\author{
Estefani Marin, Alana Ludemila de Freitas Tavares, Morgana Neves, \\ Ana Caroline Barbosa Retameiro, Lucinéia de Fátima Chasko Ribeiro, \\ Rose Meire Costa, Taciane Stein da Silva Leal e Gladson Ricardo Flor Bertolini.
}

Como citar este artigo:

MARIN, ESTEFANI.; TAVARES, ALANA LUDEMILA F.; NEVES, MORGANA.; RETAMEIRO, ANA CAROLINE B.; RIBEIRO, LUCINÉIA F. C.; COSTA, ROSE MEIRE.; LEAL, TACIANE. S. S.; BERTOLINI, GLADSON RICARDO F. Avaliação do músculo tibial anterior de ratos submetidos à um modelo de artrite reumatoide e tratados com laser de baixa potencia. Revista Saúde (Sta. Maria). 2021; 47.

\section{Autor correspondente:}

Nome: Cladson Ricardo Flor Bertolini E-mail: gladsonricardo@gmail.com Formação: Formada(o) em Fisioterapia pela Universidade Estadual de Londrina (UEL) que fica na cidade de Londrina, Paraná, Brasil. Doutor em Ciências da Saúde Aplicadas ao Aparelho Locomotor, Faculdade de Medicina de Ribeirấo Preto da Universidade de São Paulo (FMRP/ USP), Ribeirão Preto, São Paulo, Brasil.

Filiação Institucional: Universidade Estadual do Oeste do Paraná (Unioeste), Cascavel, Paraná, Brasil. Endereço: R. Universitária, n²069 Bairro: Universitário

Cidade: Cascavel

Estado: Paraná

CEP: 85819-110

Data de Submissão:

12/09/2019

Data de aceite:

09/07/202

Conflito de Interesse: Não há conflito de interesse

\section{RESUMO:}

Objetivo: avaliar os efeitos do laser de baixa potência (LBP) sobre os parâmetros histomorfométricos do músculo tibial anterior de ratos submetidos a um modelo de artrite reumatoide (AR). Métodos: foram utilizados 20 ratos machos, separados em quatro grupos $(n=5)$ : Grupo Controle (GC); Grupo Controle Laser (GCL); Grupo Lesão (GL); Grupo Lesão Laser (GLL). Para a indução do modelo de AR foram realizadas duas injeções de Adjuvante Completo de Freund. Após 24 horas da aplicação do CFA no joelho dos animais, iniciou-se o tratamento com LBP, $660 \mathrm{~nm}, 5 \mathrm{~J} / \mathrm{cm}^{2}$, em quatro pontos do joelho direito, em dias intercalados durante uma semana, totalizando quatro sessões. Sete dias após a aplicação do CFA os animais foram eutanasiados e o músculo tibial anterior direito foi coletado, pesado, cortado transversalmente e processado para lâminas histológicas, coradas em hematoxilina e eosina. Foram avaliadas características morfológicas e morfométricas. Resultados: na análise morfológica GL apresentou leves alterações morfológicas, com pequena desorganização fascicular, além de algumas fibras com perda do formato poligonal característico, também se observou aumento no número de núcleos e presença de infiltrado inflamatório. Nos animais de GLL as fibras musculares assemelharam-se às de GC, com diminuição de infiltrado inflamatório. Quanto aos dados morfométricos, observou-se diferença significativa $(p=0,004)$ na massa dos músculos pertencentes à $G L$, sendo menores que GC. Na análise da área de secção transversa $(p=0,015)$ e menor diâmetro das fibras $(p=0,019)$, os animais lesionados apresentaram menor valor. Quanto ao número de fibras $(p<0,001) G C$ apresentou menor número. Quanto ao número de núcleos, também houve diferença significativa, com GCL tendo menor número comparado a $\mathrm{GL}(\mathrm{p}=0,038)$ e $\mathrm{GLL}(\mathrm{p}=0,011)$. Ainda, $\mathrm{GC}$ apresentou maior relação núcleo/fibra comparado a $\mathrm{GCL}(p<0,001), \mathrm{GL}(p<0,001)$ e $\mathrm{GLL}(p<0,001)$. Conclusão: 0 tratamento com LBP promoveu melhora morfológica, porém não foi eficaz na total recuperação morfométrica das variáveis investigadas.

PALAVRAS-CHAVE: Terapia com Luz de Baixa Intensidade; Músculo Esquelético; Histologia.

ABSTRACT:

Aim: To evaluate the effects of low-level laser therapy (LLLT) on the histological parameters of the anterior tibial muscle of rats submitted to a rheumatoid arthritis (RA) model. Methods: 20 male rats were used, separated into four groups ( $n=5$ ): Control Group (CG); Laser Control Group (LCG); Injury Group (IG); Laser Injury Group (LIG). To induce the RA model, two injections of Freund's complete adjuvant were performed. Twenty-four hours after the application of FCA to the animals' knees, the treatment with LBP, $660 \mathrm{~nm}, 5 \mathrm{~J} / \mathrm{cm}^{2}$, was initiated in four points of the right knee, on days interspersed during one week, totaling four sessions. Seven days after the application of FCA, the animals were euthanized and the right tibialis anterior muscle was collected, weighed, cut transversely and processed into histological slides, stained in hematoxylin and eosin. Morphological and morphometric characteristics were evaluated. Results: In the IG morphological analysis, slight morphological changes were observed, with a small fascicular disorganization, in addition to some fibers with loss of characteristic polygonal shape, there was also an increase in the number of nuclei and presence of inflammatory infiltrate. In LIG the muscle fibers were similar to those of $C G$, with decreased inflammatory infiltrate. As for the morphometric data, a significant difference $(p=0.004)$ in the mass of the muscles belonging to IG was observed, being smaller than $C G$. In the analysis of the cross-sectional area $(p=0.015)$ and smaller fiber diameter $(p=0.019)$, the injured animals showed lower value. As for the number of fibers $(p<0.001) C G$ showed lower number. Regarding the number of nuclei, there was also a significant difference, with CLG having a lower number compared to IG $(p=0.038)$ and LIG $(p=0.011)$. Additionally, CG had a higher core/ fiber ratio compared to CLG $(p<0.001)$, IG $(p<0.001)$ and LIG $(p<0.001)$. Conclusion: treatment with LLLT promoted morphological improvement, but was not effective in total morphometric recovery of the variables investigated. 


\section{INTRODUÇÃO}

As doenças inflamatórias crônicas promovem incapacidade à grande parte da população mundial e geram sobrecarga econômica devido a custos diretos e indiretos ${ }^{1,2}$. No caso da Artrite Reumatoide (AR), o processo de desordem articular é gerado pelo próprio sistema imunológico devido a uma resposta inadequada, resultando na perda da qualidade de vida ${ }^{2,3}$. As lesões acometem preferencialmente articulações periféricas em simetria, avançando para a região central conforme a ascensão da cronicidade. Atinge músculos, tendões, ligamentos e vasos sanguíneos, o que produz 0 aparecimento característico de dor, fraqueza muscular, enrijecimento e deformidades que ocasiona comprometimento funcional ${ }^{4}$.

Apesar da AR produzir alterações mais acentuadas nas articulações e estruturas cartilagíneas, seu efeito sistêmico e perturbação estrutural evolutiva também agridem estruturas musculares, especialmente as que possuem relação anatômica com a região afetada, devido ao enrijecimento articular ocorre diminuição da função, e com o desuso, hipotrofia muscular e diminuição da força de contração ${ }^{5}$. O músculo tibial anterior, responsável pela dorsiflexão e inversão do pé, tangencia a articulação do joelho e transpõe o tornozelo sendo vulnerável aos efeitos deletérios da $A R^{4,6,7}$.

Devido a sua progressividade e pluralidade de estruturas acometidas, a Artrite Reumatoide demanda um tratamento criterioso cujos objetivos dependem de sua fase inflamatória e limitações funcionais ${ }^{2,8}$. Para cada qual, há necessidade de estudos acerca de terapias, exercícios e medicamentos, bem como seus efeitos sobre a AR. Para isto, são realizadas pesquisas controladas com modelos experimentais em animais para indução da AR por meio de adjuvantes ${ }^{5}$, capazes de aumentar o recrutamento de anticorpos, bem como acelerar a resposta imunológica do organismo ${ }^{4}$. O Adjuvante Completo de Freund (CFA), constituído por de óleo mineral e emulsificante da Mycobacterium butyricum, reproduz a sintomatologia característica da doença reumatoide nas articulações ${ }^{5}$. Este modelo possibilita que diferentes recursos sejam aplicados e testados no tratamento da AR, dentre eles o uso de técnicas não invasivas como a fotobiomodulação, com equipamentos como o laser de baixa potência (LBP)99.

O LBP é utilizado na prática clínica devido às suas propriedades anti-inflamatórias e eficácia na reparação de tecidos $^{10}$. O mecanismo de absorção energética ocorre por meio de proteínas presentes nas estruturas mitocondriais onde, após a interação da radiação com a célula, desencadeia-se a produção de adenosina trifosfato (ATP) juntamente a demais eventos metabólicos que tornam o processo de reparação tecidual mais rápido e eficiente ${ }^{11}$. A terapia com laser apresenta-se como boa indicação para de tratamento da AR, pois modula a resposta inflamatória tanto em estágios iniciais como tardios, mostrando efeitos de preservação das estruturas acometidas e redução do quadro álgico ${ }^{12-14}$.

No entanto, há uma lacuna com relação à repercussão do uso do LBP no tecido muscular em animais submetidos à um modelo de AR. Desta forma o objetivo do presente estudo foi avaliar os efeitos do LBP sobre os parâmetros 
histomorfométricos do músculo tibial anterior de ratos submetidos a um modelo de AR.

\section{MÉTODO}

Trata-se de uma pesquisa quantitativa, de caráter experimental, aprovada pelo Comitê de Ética da Universidade Estadual do Oeste do Paraná (UNIOESTE), realizada no Laboratório de Estudo de Lesões e Recursos Fisioterapêuticos e no Laboratório de Biologia Estrutural e Funcional (LABEF).

O grupo amostral foi composto por 20 ratos machos, da linhagem Wistar, com 15 semanas de idade, obtidos no Biotério Central da UNIOESTE e mantidos no Laboratório de Estudo de Lesões e recursos Fisioterapêuticos em caixas plásticas de polipropileno, com acesso a água e ração à vontade, temperatura controlada a $22^{\circ} \mathrm{C}$, fotoperíodo claro/ escuro de 12 horas.

Os animais foram separados aleatoriamente em quatro grupos $(n=5)$ :

- Grupo Controle (GC): sem indução com CFA ou tratamento;

- Grupo Controle Laser (GCL): sem indução com CFA, mas receberam LBP;

- Grupo Lesão (GL): animais submetidos à CFA, sem tratamento;

- Grupo Lesão Laser (GLL): com CFA e LBP.

\section{Protocolo de lesão}

A indução do modelo de AR foi realizada mediante duas injeções de CFA (Mycobacterium butyricum, 0,5 mg/ $\mathrm{ml} ; 50 \mu \mathrm{L})$. A primeira foi administrada na base da cauda do animal, via intradérmica, para imunização inicial. Após sete dias, a segunda foi administrada na articulação tibiofemoral direita. Os animais pertencentes à GC e GCL passaram pelo mesmo protocolo, porém ao invés do CFA receberam injeção com solução salina (cloreto de sódio 0,9\%) para efeito placebo.

\section{Protocolo de tratamento}

Após 24 horas da aplicação do CFA na articulação tibiofemoral dos animais pertencentes ao GLL, estes foram submetidos ao tratamento com LBP (Ibramed®). Utilizou-se o comprimento de onda de 660 nanômetros, densidade de energia para o grupo tratamento de $5 \mathrm{~J} / \mathrm{cm}^{2}$, com potência de $30 \mathrm{~mW}$, área de spot: $0,06 \mathrm{~cm}^{2}$, em quatro pontos do joelho direito (anterior, medial, lateral e posterior) (fig. 1). Energia irradiada por ponto: 0,003 J, tempo de irradiação por ponto: 10 segundos. $\mathrm{O}$ tratamento foi realizado em dias intercalados durante uma semana, totalizando quatro dias de tratamento. 
Figura 1: Aplicação da radiação laser na face anterior do joelho direito dos ratos.

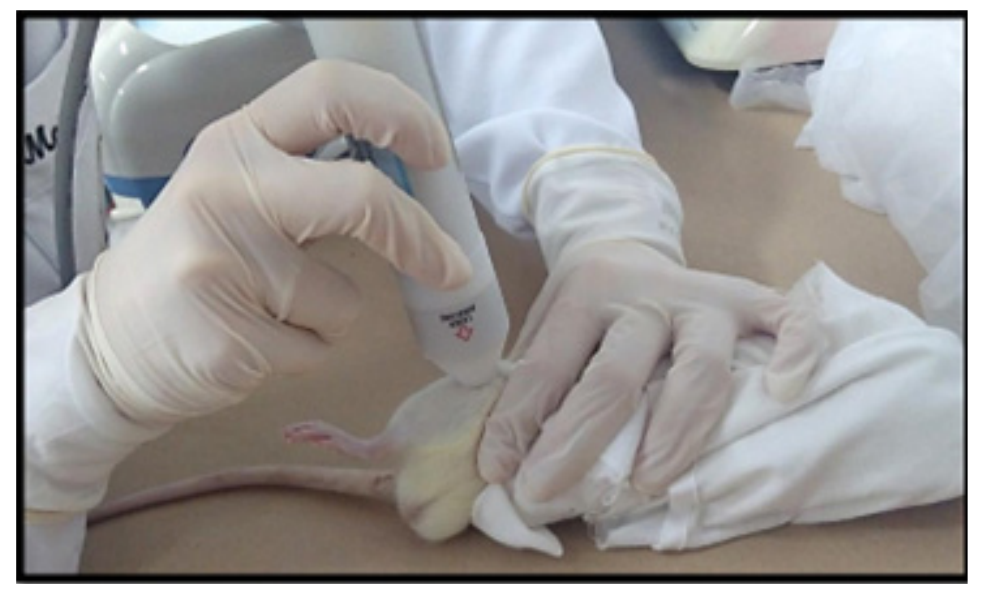

\section{Confecção das lâminas histológicas}

Sete dias após a aplicação do CFA no joelho dos animais, estes foram pesados, anestesiados com cloridrato de xilazina $15 \mathrm{mg} / \mathrm{kg}$ e cloridrato de quetamina $80 \mathrm{mg} / \mathrm{kg}$ e decapitados em guilhotina. O músculo tibial anterior do membro pélvico direito dos animais foi coletado, pesado, medido e fixado em Metacarn $(70 \%$ Metanol $+20 \%$ clorofórmio + 10\% de ácido acético glacial), durante 24 horas e posteriormente, armazenados em álcool 70\%. Posteriormente, os músculos foram processados para inclusão em parafina histológica, cortados transversalmente em $7 \mu \mathrm{m}$ de espessura em micrótomo Olympus CUT 4055, e as lâminas coradas em hematoxilina e eosina.

Para a morfologia, as lâminas foram analisadas de forma qualitativa. Já para a análise morfométrica foram realizadas fotomicrografias com aumento final de 40X e por meio do programa Image-Pro-Plus 6.0 foram analisadas 100 fibras por animal, quanto à área de secção transversa, maior e menor diâmetro, número de núcleos, número de fibras, razão de núcleos por fibra muscular, além da porcentagem de tecido conjuntivo corado com Tricrômico de Masson.

\section{Análise dos dados}

Os dados foram analisados com auxílio do programa SPSS $20.0 ®$ e apresentados pela média, seguidos de seus respectivos intervalos de confiança 95\%. Nas comparações foram utilizados os Modelos Lineares Generalizados, seguido do pós-teste Sidak. Para as variáveis de área, maior e menor diâmetro a distribuição utilizada foi Gamma, e para as demais utilizado a distribuição Normal. O nível de significância estatística considerado foi de $p<0,05$.

\section{RESULTADOS}

$\mathrm{Na}$ análise morfológica do músculo tibial anterior dos animais de GC, foi verificado aspecto normal, com presença de vasos sanguíneos, fibras organizadas em padrão fascicular e individualmente em formato poligonal, multinucleadas, 
cujos núcleos situavam-se na periferia (Fig. 2A). Morfologia semelhante foi visualizada nos animais de GCL, que não sofreram lesão, mas foram tratados com laser (Fig. 2B). Já GL apresentou leves alterações morfológicas resultantes do modelo experimental de AR (Fig. 2C), foi constatada uma pequena desorganização fascicular, além de algumas fibras com perda do formato poligonal característico. Também se observou um aparente aumento no número de núcleos, e presença de infiltrado inflamatório, verificado principalmente em regiões do epimísio e perimísio. Não foi observada alteração quanto à vascularização. Nos animais de GLL as fibras musculares assemelharam-se às de GC, sendo multinucleadas, com núcleos periféricos, poligonais, apresentando grande número de capilares sanguíneos. Também foi possível visualizar diminuição de infiltrado inflamatório quando comparado à GL (Fig. 2D).

Figura 2: Fotomicrografias do músculo tibial anterior de ratos Wistar, corte transversal, coloração em hematoxilina e eosina. Grupo Controle (A), Grupo Controle Laser (B), Grupo Lesão (C) e Grupo Lesão + Laser(D). Em A e B, organização fascicular com fibras poligonais e núcleos (Nu) periféricos. Em $\mathrm{C}$, aumento do número de núcleos $(\mathrm{Nu})$ e infiltrado inflamatório $\left(^{*}\right)$. Em D, núcleos periféricos $(\mathrm{Nu})$, pequena presença de infiltrado inflamatório $\left.{ }^{*}\right)$ e vasos sanguíneos $(\mathrm{Vs})$.
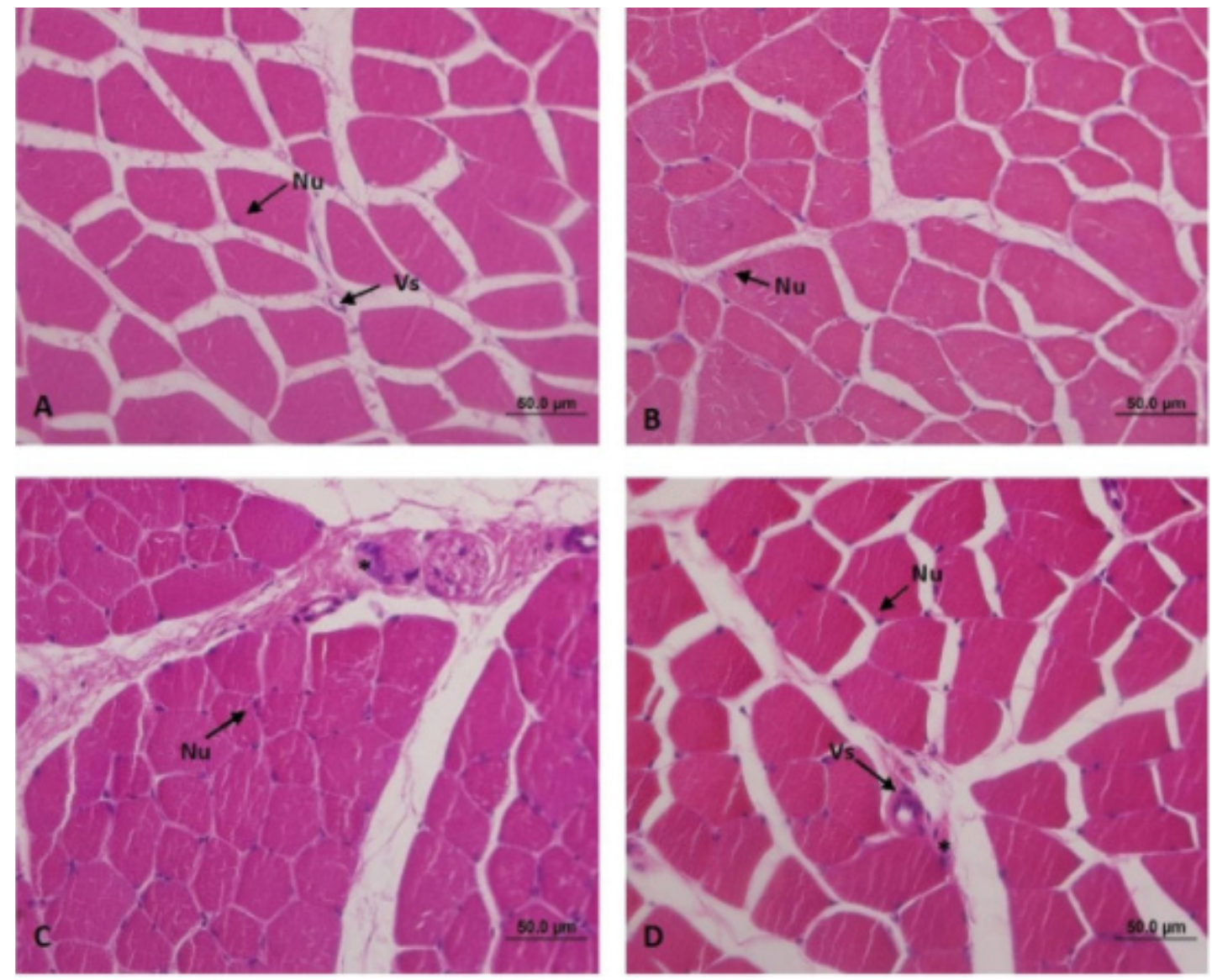

Quanto aos dados morfométricos, observou-se, diferença significativa [Wald $X 2(3)=13,32 ; p=0,004$ ] na massa dos músculos pertencentes à $G L$, sendo menores quando comparados à $G C(p=0,19)$ e $G C L(p=0,007)$. Quanto ao comprimento do músculo, não houve diferença significativa [Wald X2(3)=2,77; $p=427$ ] (Tabela 1). 
Tabela 1: Média e seus respectivos intervalos de confiança $95 \%$ dos valores do peso e comprimento muscular do músculo tibial anterior de ratos Wistar.

\begin{tabular}{|l|c|c|c|c|}
\hline & GC & GCL & GL & GLL \\
\hline Massa (g) & $0,60[0,5-0,6]^{2}$ & $0,47[0,4-05]^{\text {bc }}$ & $0,61[0,5-0,6]^{2}$ & $0,54[0,4-0,6]^{\text {zbc }}$ \\
\hline Comprimento $(\mathbf{m m})$ & $23,40[21,3-25,5]^{2}$ & $21,80[19,7-23,9]^{2}$ & $22,6[20,5-24,7]^{2}$ & $21[18,9-23,1]^{2}$ \\
\hline
\end{tabular}

Legenda: GC- Grupo Controle; GCL- Grupo Controle Laser; GL- Grupo Lesão; GLL- Grupo Lesão + Laser. Letras diferentes significam valores estatisticamente diferentes $(p \leq 0,05)$.

$\mathrm{Na}$ análise da área de secção transversa [Wald X2(3)=10,452; $p=0,015]$ e menor diâmetro das fibras [Wald $X 2(3)=5,103 ; p=0,019]$, observou-se diferença estatisticamente significativa entre $G C$ e $G L(p<0,05)$, em que os animais lesionados apresentaram menor valor. Já para o maior diâmetro [Wald $X 2(3)=5,103 ; p=164]$, não houve diferença significativa entre os grupos. Quanto ao número de fibras [Wald X2(3)=39,33; $p<0,001$ ], houve diferença significativa entre GC, GL e GLL ( $p<0,001)$, sendo que GC apresentou menor número de fibras. De forma semelhante, GCL apresentou número significativamente menor de fibras quando comparado à $G L(p=0,004)$ e $G L L(p<0,001)$ (Tabela 2).

No que tange ao número de núcleos, também houve diferença significativa [Wald $X 2(3)=14,500 ; p=0,002$ ]. GCL apresentou menor número comparado com GL $(p=0,038)$ e $G L L(p=0,011)$. Ainda, houve diferença significativa [Wald $X 2(3)=39,45 ; p<0,001)$ para a relação núcleo/fibra, em que $G C$ apresentou maior relação comparado a $G C L(p<0,001)$, GL ( $p<0,001)$ e GLL ( $p<0,001)$. Quanto ao tecido conjuntivo, não houve diferença significativa [Wald $X 2(3)=4,27 ; p=0,233)$ entre os grupos (Tabela 2).

Tabela 2: Média e seus respectivos intervalos de confiança 95\% da área de secção transversa, maior diâmetro, menor diâmetro, em $\mu \mathrm{m}$, número de fibras e núcleos, além da porcentagem de tecido conjuntivo (\%) das fibras do músculo tibial anterior de ratos Wistar.

\begin{tabular}{|c|c|c|c|c|}
\hline & GC & GCL & GL & GLL \\
\hline Área de Secção & & & & \\
\hline $\begin{array}{c}\text { Transversa } \\
\left(\boldsymbol{\mu m}^{2}\right)\end{array}$ & $83,08[69,1-99,8]^{\circ}$ & $67,56[56,2-81,1]^{\text {bे }}$ & $54,74[45,5-65,7]^{\circ}$ & $72,88[60,6-87,5]^{\circ b}$ \\
\hline $\begin{array}{c}\text { Menor diâmetro } \\
\mathbf{( \mu m )}\end{array}$ & $7,76[7,1-8,4]^{\circ}$ & $7,06[6,4-7,7]^{\text {b }}$ & $6,38[5,8-6,9]^{\circ}$ & $7,28[6,6-7,9]^{\circ b}$ \\
\hline
\end{tabular}




\begin{tabular}{|c|c|c|c|c|}
\hline $\begin{array}{l}\text { Maior diâmetro } \\
\qquad(\mu \mathrm{m})\end{array}$ & $11,59[10,3-12,9]^{\circ}$ & $10,91[9,7-12,2]^{\prime}$ & $9,79[8,7-10,5]^{\mathrm{s}}$ & $11,30[10,1-12,6]^{\mathrm{s}}$ \\
\hline $\begin{array}{c}\text { Número de } \\
\text { fibras }\end{array}$ & $17,46[15,2-19,6]^{3}$ & $19,28[17,10-21,4]^{3}$ & $24,6[22,4-26,8]^{b}$ & $25,68[23,05-27,8]^{\circ}$ \\
\hline $\begin{array}{l}\text { Número de } \\
\text { núcleos }\end{array}$ & $30,64[27-34,2]^{t b}$ & $29,26[25,6-32,8]^{\circ}$ & $36,4[32,7-40]^{\circ}$ & $37,42[33,7-40]^{\prime}$ \\
\hline $\begin{array}{c}\text { Razão } \\
\text { núcleo/fibra }\end{array}$ & $1,92[1,81-2]^{\mathrm{E}}$ & $1,51[1,4-1,6]^{b}$ & $1,51[1,4-1,6]^{b}$ & $1,54[1,4-1,6]^{b}$ \\
\hline $\begin{array}{c}\text { Tecido } \\
\text { conjuntivo } \\
(\%)\end{array}$ & $1,72[1,4-2]^{\circ}$ & $1,55[1,2-1,8]^{\mathrm{s}}$ & $1,9039[1,6-2,2]^{\prime}$ & $1,50[1,2-1,8]^{\circ}$ \\
\hline
\end{tabular}

Legenda: GC- Grupo Controle; GCL- Grupo Controle Laser; GL- Grupo Lesão; GLL- Grupo Lesão + Laser. Letras diferentes significam valores estatisticamente diferentes $(p \leq 0,05)$.

\section{DISCUSSÃO}

O presente estudo avaliou características morfológicas e morfométricas do músculo tibial anterior, em um modelo de $A R$, frente à terapia com LBP, o qual mostrou em algumas variáveis discreta melhora frente ao placebo.

Devido à complexidade e problemas éticos para a realização de análises invasivas em humanos com AR, pesquisas utilizando modelos experimentais são úteis, principalmente quanto ao teste de algumas modalidades terapêuticas. Para isso, o modelo induzido por Adjuvante Completo de Freund (CFA) vem sendo muito utilizado na mimetização das manifestações dessa inflamação ${ }^{5}$. No presente estudo, esse modelo repercutiu em alterações morfológicas e morfométricas no músculo esquelético. Na análise da morfologia do músculo tibial anterior dos animais pertencentes à GL, observou-se atrofia das fibras musculares, desorganização fascicular, aumento do número de núcleos e presença de infiltrado inflamatório, que podem ser decorrentes do maior índice de moléculas de adesão no endotélio vascular, facilitando o processo de migração das células inflamatórias para o local15.

Quanto à vascularização, não houve diferença entre GC e GL, visto que ambos apresentaram boa vascularização, tal achado corrobora com o relatado por Sandoo et al. ${ }^{16}$, que citam inexistir associação entre a inflamação sistêmica resultante da AR e prejuízos na vascularização. Quanto à morfologia dos animais pertencentes à GLL, observou-se uma 
reversão dos efeitos deletérios do modelo de AR. Assis et al. ${ }^{17}$ ao estudarem a cartilagem articular de ratos submetidos a um modelo experimental de osteoartrite, relatam que a irradiação do laser associada a exercícios de corrida em esteira previnem a degeneração da cartilagem e modulação do processo inflamatório.

Em virtude do seu caráter autoimune, a AR resulta em uma desordem inflamatória que atinge órgãos e tecidos, gerando perda de função ${ }^{18}$. Diversos autores ${ }^{19-21}$ apontam para a perda de massa muscular e incapacidade funcional em pacientes com AR, sendo difícil a restauração deste tecido nestes $\operatorname{casos}^{22}$. Tendo em vista a vulnerabilidade do músculo tibial anterior aos efeitos da $\mathrm{AR}^{6}$, o presente estudo observou uma diminuição no peso do músculo tibial anterior dos animais induzidos. Ainda quanto às propriedades morfométricas, a presente pesquisa apresentou que animais com AR tiveram diminuição da área de secção transversa, menor diâmetro e razão núcleo/fibra.

No que se refere as modalidades de tratamento da $A R$, há uma pluralidade de intervenções visando a melhora da saúde em geral ${ }^{23,24}$. Sendo que o LBP apresenta efeitos positivos no alívio dos sintomas em pacientes com AR 25. O tratamento emite uma radiação que penetra nos tecidos, absorvida no Citocromo $\mathrm{C}$ oxidase, responsável pela transferência de elétrons do Citocromo C para a molécula de oxigênio, auxiliando na produção de adenosina trifosfato (ATP), contribuindo para uma sequência de eventos responsáveis pelo reparo tecidual26,27. No presente estudo, 0 tratamento com tal recurso apesar de não ter sido diferente do grupo apenas lesão, mostrou-se semelhante aos grupos controle, indicando que estava em processo de recuperação de seu trofismo. Considerando-se que essa terapia não foi eficaz em algumas das variáveis analisadas, visto o observado com respeito ao número de fibras e núcleos, sugere-se novos estudos abordando diferentes comprimentos de onda e alterações na dosimetria utilizada.

\section{CONCLUSÃO}

O modelo de artrite reumatoide repercutiu em alterações morfológicas e morfométricas no músculo tibial anterior de ratos Wistar, e o tratamento com laser de baixa potência promoveu melhora morfológica, porém não foi eficaz na total recuperação morfométrica das variáveis investigadas.

\section{AGRADECIMENTOS}

Ao CNPq pela cessão de bolsa de iniciação científica.

\section{REFERÊNCIAS}

1. Emery P, Burmester GR, Naredo E, Zhou Y, Hojnik M, Conaghan PG. Design of a phase IV randomised, 
assessing the impact of residual inflammation detected via imaging techniques, drug levels and patient characteristics on the outcome of dose tapering of Adalimumab in clinical remission Rheumatoid Arthritis (RA) patients. BMJ Open. 2018;8(2):e019007.

2. Fröohlich JK, Stein T, da Silva LA, Biavatti MW, Tonussi CR, Lemos-Senna E. Antinociceptive and anti-inflammatory activities of the Jatropha isabellei dichloromethane fraction and isolation and quantitative determination of jatrophone by UFLC-DAD. Pharm Biol. 2017;55(1):1215-22.

3. Hung L-H, Wu C-H, Lin B-F, Hwang LS. Hyperimmune colostrum alleviates rheumatoid arthritis in a collagen-induced arthritis murine model. J Dairy Sci. 2018;101:3778-87.

4. Souza MCP de, Lourenço JRFL, Dellalibera-Joviliano R. Imunomodulação em modelo experimental de artrite reumatóide submetido ao tratamento de complexo de paládio e natação. Rev Bras Prescrição e Fisiol do Exerc. 2017;11(67):439-46.

5. Gomes RP, Bressan E, Silva TM da, Gevaerd M da S, Tonussi CR, Domenech SC. Efeitos de um minuto e dez minutos de deambulação em ratos com artrite induzida por adjuvante completo de Freund sobre os sintomas de dor e edema. Rev Bras Reumatol. 2014;54(2):83-9.

6. Pereira ALN, Pastori M dos S, Leite N dos S de ML, Pereira TR, Kawamoto TT, Ferreira KK, et al. Tenossinovite de tornozelo em artrite reumatoide : avaliação clínica e ultrassonográfica. Rev Fac Ciênc Méd Sorocaba. 2016;18(3):1821.

7. Sonnery-Cottet B, Mogos S, Thaunat M, Archbold P, Fayard J-M, Freychet B, et al. Proximal tibial anterior closing wedge osteotomy in repeat revision of anterior cruciate ligament reconstruction. Am J Sports Med. 2014;42(8):187380.

8. Negrei C, Bojinca V, Balanescu A, Bojinca M, Baconi D, Spandidos DA, et al. Management of rheumatoid arthritis: Impact and risks of various therapeutic approaches (Review). Exp Ther Med. 2016;11(4):1177-83.

9. Paolillo FR, Paolillo AR, João JP, Frascá D, Duchêne M, João HA, et al. Ultrasound plus low-level laser therapy for knee osteoarthritis rehabilitation: a randomized, placebo-controlled trial. Rheumatol Int. 2018;38(0):785-93.

10. Dahmardehei M, Kazemikhoo N, Vaghardoost R. Effects of low level laser therapy on the prognosis of split-thickness skin graft in type 3 burn of diabetic patients: a case series. Lasers Med Sci. 2016;31(1):497-502.

11. Ginani F, Soares DM, Augusto C, Barboza G. Effect of low-level laser therapy on mesenchymal stem cell proliferation: a systematic review. Lasers Med Sci. 2015;30(8):2189-94.

12. Alves ACA, De Carvalho PDTC, Parente M, Xavier M, Frigo L, Aimbire F, et al. Low-level laser therapy in different stages of rheumatoid arthritis: A histological study. Lasers Med Sci. 2013;28(2):529-36. 
13. Carlos FP, Paula M De, Costa MS, Zamuner SR. Protective effect of low-level laser therapy (LLLT) on acute zymosan-induced arthritis. Lasers Med Sci. 2014;29(2):757-63.

14. Ip D. Does addition of low-level laser therapy (LLLT) in conservative care of knee arthritis successfully postpone the need for joint replacement? Lasers Med Sci. 2015;30(9):2335-9.

15. Teixeira RC de A, Gabriel Júnior A, Martino MC de, Martins LC, Lopes AC, Tufik S. Marcadores de ativação endotelial e auto-anticorpos na artrite reumatóide. Rev Bras Reumatol. 2007;47(6):411-7.

16. Sandoo A, Zanten JJCSV Van, Metsios GS, Carroll D, Kitas GD. Vascular function and morphology in rheumatoid arthritis: a systematic review. Rheumatology. 2011;50(11):2125-39.

17. Assis L, Milares LP, Almeida T, Tim C, Magri A, Fernandes KR, et al. Aerobic exercise training and low-level laser therapy modulate inflammatory response and degenerative process in an experimental model of knee osteoarthritis in rats. Osteoarthr Cartil. 2016;24(1):169-77.

18. Cooney JK, Law R-J, Matschke V, Lemmey AB, Moore JP, Ahmad Y, et al. Benefits of exercise in rheumatoid arthritis. J Aging Res. 2011 Feb;2011:681640.

19. Matschke V, Murphy P, Lemmey AB, Maddison P, Thom JM. Skeletal muscle properties in rheumatoid arthritis patients. Med Sci Sport Exerc. 2010;42(12):2149-55.

20. Matschke V, Murphy P, Lemmey AB, Maddison PJ, Thom JM, Matschke V, et al. Muscle quality, architecture, and activation in cachectic patients with rheumatoid arthritis. J Rheumatol. 2010;37(2):282-4.

21. Santana FS De, Nascimento C, Paulo J, Freitas M De, Miranda RF, Muniz LF, et al. Assessment of functional capacity in patients with rheumatoid arthritis: implications for recommending exercise. Rev Bras Reumatol. 2014;54(5):378-85.

22. Teixeira V de ON, Filippin LI, Xavier RM. Mecanismos de perda muscular da sarcopenia. Rev Bras Reum. 2012;52(2):247-59.

23. Camacho-Bautista K, Orjuela-Rodríguez A, Vargas-Pinilla O. Intervención fisioterapéutica en el manejo de pacientes con artritis reumatoide: una revisión sistemática. Fisioterapia. 2017;39(4):174-80.

24. Faganello L, Colet $C$ de F. Uso de medicamentos por pacientes com artrite reumatóide em um município do interior do Rio Grande do Sul. Saúde (Santa Maria). 2018:44(1):1-14.

25. Brosseau L, Welch V, Ga W, R DB, Gam A, Harman K, et al. Low level laser therapy (Classes I , II and III) for treating rheumatoid arthritis. Cochrane Database Syst Rev. 2005;19(4):CD002049.

26. Smith KC. Molecular targets for low level light therapy. Laser Ther. 2010;19(3):135-42.

27. Karu TI. Multiple roles of cytochrome c oxidase in mammalian cells under action of red and IR-A radiation. IUBMB Life. 2010;62(8):607-10. 